\title{
NAMED ENTITY RECOGNITION AND INFORMATION EXTRACTION FOR ARABIC MEDICAL TEXT
}

\author{
Jaafar Hammoud, Natalia Dobrenko and Natalia Gusarova \\ ITMO University \\ Sankt-Petersburg 197101, Russia
}

\begin{abstract}
The article discusses the possibilities of solving NER (Named Entity Recognition) problem for medical texts in Arabic with limited availability of labeled datasets, as well as computational and specialized linguistic resources. To overcome them, it is proposed to use recurrent neural networks. In our experiments, we used "BERT-Base, Multilingual Cased" from Google and Pooled-GRU with Multi-lingual Universal Sentence Encoder (MUSE) from Facebook. Each network was fine-tuned with our dataset.

The used dataset was obtained from three medical volumes issued by Arabic Encyclopedia. We experimentally evaluated the effectiveness of tuned models on real NLP (Natural Language Processing) task - medical entities recognition from the Arabic Medical Encyclopedia and obtained encouraging results.
\end{abstract}

\section{KEYWORDS}

Natural Language Processing, Named Entity Recognition, Arabic, e-Health

\section{INTRODUCTION}

E-health has been recognized as a cost-effective and reliable form of developing health systems and services, including in relatively low-income countries and in regions where health coverage is inadequate. However, language barriers often become a real obstacle to the introduction of advanced E-health technologies in real healthcare practice in such countries. An Indication in this sense is Arabic. The gap between it and other languages such as English and European Union languages is still huge because of such reasons like orthographic ambiguity and inconsistency, morphological inflections, word order freedom, dialectal variation, and lack of adequate dataset (Maloney, 1998). These circumstances complicate the direct application of NLP methods with these languages, which are mainly developed and verified on English sources.

The NER task is one of the primary keys that used in texts processing. Many NLP applications such as question answering, information retrieval, and machine translation require a NER system to enhance their results. In 1995, at the Sixth Message Understanding Conference, the concept of the NER was first introduced, which aims to develop a new method to improve the process of information extracting. NER works to extract predefined entities from an unstructured text that can include person names, location names, organizations, percentages, temporal expressions, money expressions, and numerical expression (Alanazi, 2017).

There are two common groups of methods for dealing with entities extraction and recognition. The first uses linguistics grammar-based techniques. As a rule, these methods obtain better accuracy, but they are language dependent and require months of work by experienced computational linguists (Nothman, 2013).

The second group is based on statistical models and uses traditional machine learning in combination with the latest techniques. The biggest benefit to be gained from these methods is that they are context sensitive. Accordingly, they have advantages when processing texts in languages with complex context-sensitive grammar, such as Arabic. But they need a large amount of manually annotated training data, and the more advanced algorithms require significant computational resources.

The article discusses the possibilities of solving NER problem for medical texts in Arabic with limited availability of labeled datasets, as well as computational and specialized linguistic resources. 
Consequently, In the article, we identified the following tasks

- Create a dataset from available medical texts in Arabic.

- Choose NN models that obtaining competitive performance when solving the NER problem and fine-tune them in the conditions of the above limitations.

- Experimentally evaluate the effectiveness of tuned models on real NLP tasks.

\section{RELATED WORKS}

Through a literary review of past and related works, NER systems in Arabic language are limited to political and economic fields, and most of the texts used were based mainly on news agencies. Moreover, NER systems were rarely used on any Arabic medical documents.

Until 2012, researchers focused on the rule-based approach, many articles used own and small corpus or ANERcorp to recognize person names, organizations, locations, date, and numerical expression (Al-Shalabi, 2009; Elsebai, 2009; Al-Jumaily, 2012; Shihdaeh, 2012; Zaghouani, 2012). To recognize the crime type, location, and nationality researchers used a rule-based approach too on Arabic Crime News Report Corpus (ANCRC) (Alruily, 2012).

On another hand, to recognize (person names, locations, organization, and miscellaneous) researchers used maximum entropy on ANERcorp (Benajiba, 2007), used conditional random fields on ANERcorp (Benajiba, 2008; Morsi, 2013) and ACE (2005) (Abdul-Hamid, 2010), and used support vector machine on UPVCorpus and ACE (2003-2005) (Benajiba, 2008; Koulali, 2012). In (Helwe, 2019) researchers used deep co-learning, and in (Ali, 2019) they boosted Arabic named entity recognition with a multi-attention layer.

In (Albukhitan, 2013) researches introduced an Arabic semantic annotation tool with integrated information extraction for food, nutrition, and health. In (Dudchenko, 2013) researchers used the Bayesian Belief Network (BBN) to extract disease name, symptoms, treatment methods, and diagnosis methods.

In (Salah, 2017) authors used two approaches, the first was the traditional ML with CRF and the second was a DNN model which used Bi-LSTM-CRF. In (Shahina, 2019) researchers used different versions of RNN cells for NER problem (e.g. Bi-LSTM, Bi-GRU, and Bi-RNN) on ANERcorp dataset.

\section{DATASET}

First, we have obtained the dataset from three medical volumes issued by the Arabic Encyclopedia in the Syrian Arab Republic, available as text on the Internet through the encyclopedia site ${ }^{1}$, using the Scrapy ${ }^{2}$ library, a free and open-source web crawling framework written in Python. The three volumes are (Respiratory System Diseases, Cardiovascular Diseases, and Skin Diseases). In Table 1, the number of articles in each volume and the number of sentences are shown.

Table 1. Source characteristics

\begin{tabular}{lcc}
\hline Volume & Number of articles & Number of sentences \\
\hline Respiratory System Diseas & 28 & 6691 \\
Cardiovascular Diseases & 33 & 9464 \\
Skin Diseases & 22 & 5921 \\
\hline
\end{tabular}

Articles are relatively long in these volumes, and one article may contain more than one partial article within it. For example, an article that talks about electrocardiograms mentions all types of electrocardiograms within separate paragraphs, and we can consider that each paragraph of the text is a special article. But in our study and because of the use of the BERT model for training, we care more about the concept of a sentence than the article concept.

\footnotetext{
${ }^{1}$ http://arab-ency.com.sy

${ }^{2}$ https://scrapy.org/
} 
The annotation of entities (disease name, organ name, disease symptoms, and drug name) were done manually using the IOB rule by some Arabic medical students at Arab universities. Then, the English and French terms were removed from the text and only the Arabic text was retained. Figure 1 is a screenshot of the data format.

\begin{tabular}{|c|c|}
\hline B-DISEASE الربو & O Oسبب \\
\hline O حالة & O هذه \\
\hline O التهابية & O الحالة \\
\hline O مزمنة & O الالتهابية \\
\hline O & B-SYMPTOM فرط \\
\hline B-ORGAN الطرق & استثارة I-SYMPTOM \\
\hline & اقصبية I-SYMPTOM \\
\hline |-ORGAN التنفسية |-ORG & O لعوامل \\
\hline O تعتمد O & O منبهة \\
\hline O على & O ينجم \\
\hline B-ORGAN الخلايا & ( \\
\hline I-ORGAN البدينة I-ORAN & B-SYMPTOM أزيز \\
\hline $\mathrm{O}$ & إنوبي \\
\hline O محبات & I-SYMPTOM نفسيق \\
\hline O الحامض & B-SYMPTOM وسعال \\
\hline O واللمفيات & Oأَكثره \\
\hline O المساعدة & O في \\
\hline
\end{tabular}

Figure 1. Screenshot from the dataset

The Arabic Medical Encyclopedia consists of several volumes, each article in these volumes is written by a different physician, so there is no unified structure for articles, and in a large number of articles, the symptoms are mentioned in the context of the text and not in a special paragraph.

There is no unified tag for every new paragraph in the article, titles of some paragraphs tagged as "strong", others as "h2", etc. For these reasons, most of the pre-processing was done manually.

Also, one of the problems that we encountered while working with text is sentence length, especially sentences for symptoms of disease and difficulty in determine the appropriate sentence, we illustrate that in the following example Figure 2.

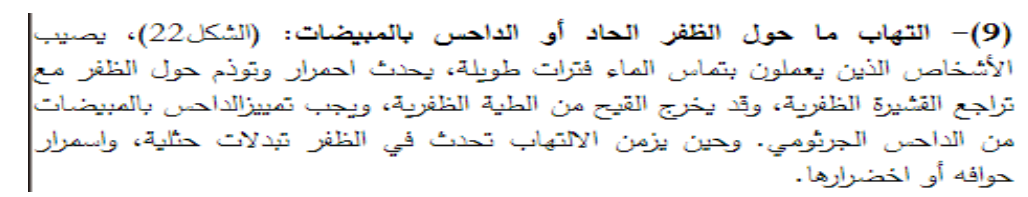

Figure 2. An example that shows how difficult it is to choose a sentence that describes a symptom of an illness. Translation of figure 2: Inflammation around the sharp nail or paronychia with candidiasis affects people who work in contact with water for long periods. Redness and swelling occurs around the nail as the nail scales retract and pus may come out of the nail fold. The paronychia of Candida must be distinguished from bacterial paronychia. When the inflammation is chronic, inflammation in the nail changes in its induction and darkening of its edges or being green

We notice the interconnectedness of the sentence together, and we can also choose the symptoms in more than one way. This affects the training of the model, for example:

- Redness and edema around the nail

- Redness

- edema

- nail plate retracts

Figure 3 also illustrates the problem of overlapping more than one entity together. 


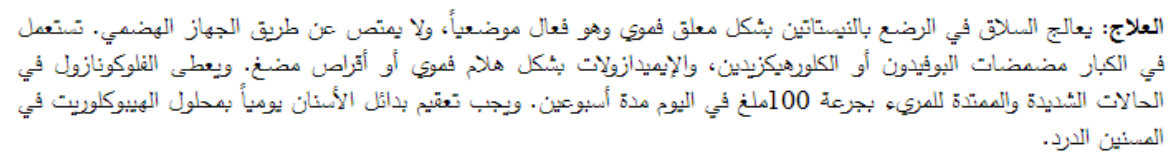

Figure 3. Overlapping more than one entity together. Translation of figure 3: Treatment: Slave is treated in infants with nystatin in the form of an oral suspension, which is effective locally and not absorbed by the digestive system. It is used in adults as povidone or chlorhexidine and imidazole in the form of an oral gel or chewable tablets. Fluconazole is given in severe and prolonged cases in a dose of $100 \mathrm{mg}$ per day for two weeks and sterilization alternatives should be sterilized Teeth daily with hypochlorite solution

The sentence is composed of the names of some medicines, and at the same time the sequence of words represents a treatment.

The BERT model requires for text processing (see Section 4) a special format for the data before working with the model. The sentence must start with the [CLS] token, while the [SEP] token is added at the end of each sentence. Thus, for Arabic tokenization, we used the BERT tokenizer which uses WordPiece (Wu, 2016). After that, from the token vocabulary file that BERT is provided, every token is mapped to an index.

\section{CHOSEN NNS AND THEIR FINE-TUNING}

First, we fine-tuned BERT model (Devlin, 2018) published by researchers at Google AI Language ${ }^{3}$ with our dataset. This pre-trained model has raised the interest of researchers in a field of machine learning and has provided distinctive results in a wide range of NLP applications. BERT uses a mechanism that learns contextual relations between words in a text by using the transformer. The transformer includes two separate mechanisms, the first is an encoder that reads the text as input and a decoder that produces a prediction for the input. $15 \%$ of the words in each sequence are replaced with a [MASK] token then the model attempts to predict the original value of the masked words by using the context provided by non-masked words. Using BERT, the NER model can be trained by feeding the output vector of each token into a classification layer that predicts the NER label. In NER, two popular approaches:

1. A probabilistic graphical model called CRF (Conditional Random Field), in which the token is labeled considering the context.

2. Multi-class Classification-based: each token in the sentence will get a label, so we can treat our process as a multi-class classification problem, and that what we did in our work.

We used the library deployed by hugginface ${ }^{4}$ and called transformers, this library includes pre-trained models for NLP problems that achieve state-of-the-art results. Our dataset was separated into two groups ( $80 \%$ as training data) and (20\% as testing data) and then with the dataset we fine-tuned a pre-trained model called "BERT-Base, Multilingual Cased" which supports 104 languages, and contains 12 layers, 768 hidden, 12 heads, and 110 million parameters. For the purposes of fine-tuning, we chose the following values: Batch size $=32$; Learning rate $($ Adam $)=2 \mathrm{e}-5$; Number of epochs $=4$; The epsilon parameter eps $=1 \mathrm{e}-8$.

The BERT built on pre-training contextual representations that including the Transformer (Vaswani, 2017), Semi-supervised Sequence Learning (Dai, 2015), the OpenAI transformer (Radford, 2018), ELMo (Peters, 2018), and ULMFiT (Howard, 2018).

BERT was pre-trained on a large corpus of unlabeled text which includes the entire Wikipedia and a book corpus and what makes BERT unique compared to the rest of models that it is first deeply bidirectional, unsupervised language representation, and pre-trained using only a plain text corpus, on other hand, BERT was first successfully pre-train bidirectional attempt in a deep neural network. While traditional context-free models generate a single word embedding for each word, BERT would represent based on both the left and the right context.

The two biggest positive points in using the BERT model are:

- Quicker Development: the authors recommend only 2-4 epochs of training for fine-tuning BERT on a specific NLP task.

\footnotetext{
${ }^{3}$ https://research.google/teams/language

${ }^{4}$ https://github.com/huggingface/transformers
} 
- Less Data: Because of the pre-trained weights BERT allows us to fine-tune our task on a small dataset.

As researchers refer and because the way that BERT was trained by, we can use BERT for many NLP tasks like question answering system, text classification (and in our work, we treat the task of NER as a multi-class classification as mentioned before), part-of-speech tagging, etc.

Second, in (Al-Smadi, 2020) researchers introduced a new model for working with ANER (Arabic named entity recognition)

This model consists of 6 layers and we have made some adjustments to it to fit our dataset

1. Text embedding: the model uses "Multi-lingual Universal Sentence Encoder (MUSE)" introduced by Facebook (Cer, 2018).

2. Bi-Directional-GRU: with a relatively training time faster than Long Short-Term Memory (LSTM) an enhanced version of RNN is used in this model.

3. Pooling layer: in this layer to extract the discriminative features of the input text the model used the Global Average Pooling and Global Max Pooling.

4. Concatenation layer: to keep all the possible discriminative features in this layer and after computing both values of GAP and GMP concatenate them in a single vector.

5. Fully connected network: to learn the discriminative features from the previous layer and with ReLU activation function a Dense layer with 512 fully connected neurons.

6. Classification layer work with IOB framework.

\section{RESULTS AND DISCUSSION}

We measure the accuracy of the model by using f1 score:

The Precision computed by the following equation:

$$
F 1=2 * \frac{\text { Precision } * \text { Recall }}{\text { Precision + Recall }}
$$

$$
\text { Precision }=\frac{T P}{T P+F P}
$$

and the Recall computed by the following equation:

$$
\text { Recall }=\frac{T P}{T P+F N}
$$

where TP denotes to True Positive, FP False Positive, and FN False Negative.

Table 2. F1 Score for every entity

\begin{tabular}{lcccc}
\hline & disease name & organ name & $\begin{array}{c}\text { disease } \\
\text { symptoms }\end{array}$ & drug name \\
\hline BERT & 87.7102 & 86.3021 & 69.8519 & 77.4986 \\
\hline Pooled-GRU & 88.9133 & 87.5129 & 72.6125 & 76.5148 \\
\hline
\end{tabular}

All previous research that studied this issue on Arabic, relied relatively on small datasets, in addition to that, the entities were limited to the names of people, places, and organizations. In the medical field, researchers whose we were able to review their works focused on one disease. such as cancer.

However, the results were very promising, compared to the results found for the same problem in languages such as English and French.

\section{CONCLUSION}

The tasks posed in the article have been successfully solved. Namely:

- We created a dataset from available medical texts in Arabic. 
- We have chosen two NN models of recurrent type which obtained competitive performances when solving the NER problem.

- We experimental evaluated the effectiveness of tuned models on real NLP tasks. The models provide good results compared to the existing level.

As future work, we suggest applying the rest of the Transformers models that have achieved state-of-the-art results, such as (BERT, GPT-2, RoBERTa, XLM, DistilBert, XLNet, CTRL, etc) on the Arabic for various tasks (Text classification, Text summarization, Question-answering systems, etc.)

\section{ACKNOWLEDGEMENT}

This work was financially supported by Russian Science Foundation, Grant 19-19-00696.

\section{REFERENCES}

Abdul-Hamid, A., \& Darwish, K. (2010, July). Simplified feature set for Arabic named entity recognition. In Proceedings of the 2010 Named Entities Workshop (pp. 110-115). Association for Computational Linguistics.

Alanazi, S. (2017). A named entity recognition system applied to Arabic text in the medical domain (Doctoral dissertation, Staffordshire University).

Albukhitan, S., \& Helmy, T. (2013, January). Automatic Ontology-based Annotation of Food, Nutrition and Health. Arabic Web Content. In ANT/SEIT (pp. 461-469).

Ali, M. N. A., et al. (2019). Boosting Arabic named-entity recognition with multi-attention layer. IEEE Access, 7, 46575-46582.

Al-Jumaily, H., et al. (2012). A real time Named Entity Recognition system for Arabic text mining. Language resources and evaluation, 46(4), 543-563.

Alruily, M. (2012). Using text mining to identify crime patterns from arabic crime news report corpus.

Al-Shalabi, R., et al. (2009, March). Proper noun extracting algorithm for arabic language. In International conference on IT, Thailand.

Al-Smadi, M., et al. (2020). Transfer Learning for Arabic Named Entity Recognition With Deep Neural Networks. IEEE Access, 8, 37736-37745.

Benajiba, Y., \& Rosso, P. (2007, December). ANERsys 2.0: Conquering the NER Task for the Arabic Language by Combining the Maximum Entropy with POS-tag Information. In IICAI (pp. 1814-1823).

Benajiba, Y., \& Rosso, P. (2008, May). Arabic named entity recognition using conditional random fields. In Proc. of Workshop on HLT \& NLP within the Arabic World, LREC (Vol. 8, pp. 143-153).

Benajiba, Y., et al. (2008). Arabic named entity recognition: An svm-based approach. In Proceedings of 2008 Arab International Conference on Information Technology (ACIT) (pp. 16-18). Amman, Jordan: Association of Arab Universities.

Cer, D., et al. (2018). Universal sentence encoder. arXiv preprint arXiv:1803.11175.

Dai, A. M., \& Le, Q. V. (2015). Semi-supervised sequence learning. In Advances in neural information processing systems (pp. 3079-3087).

Devlin, J., et al. (2018). Bert: Pre-training of deep bidirectional transformers for language understanding. arXiv preprint arXiv:1810.04805.

Dudchenko, A., et al. (2019). Extraction from Medical Records. Stud. Health Technol. Inform, 261, 62-67.

Elsebai, A. (2009). A rules based system for named entity recognition in modern standard Arabic (Doctoral dissertation, University of Salford).

Habash, Nizar Y. (2010). Introduction to Arabic natural language processing. Synthesis Lectures on Human Language Technologies 3.1, pp 1-187.

Helwe, C., \& Elbassuoni, S. (2019). Arabic named entity recognition via deep co-learning. Artificial Intelligence Review, 52(1), 197-215.

Howard, J., \& Ruder, S. (2018). Universal language model fine-tuning for text classification. arXiv preprint arXiv:1801.06146.

Koulali, R., \& Meziane, A. (2012, November). A contribution to Arabic named entity recognition. In 2012 Tenth International Conference on ICT and Knowledge Engineering (pp. 46-52). IEEE. 
Maloney, J., \& Niv, M. (1998). TAGARAB: a fast, accurate Arabic name recognizer using high-precision morphological analysis. In Computational Approaches to Semitic Languages.

Morsi, A., \& Rafea, A. (2013, May). Studying the impact of various features on the performance of Conditional Random Field-based Arabic Named Entity Recognition. In 2013 ACS International Conference on Computer Systems and Applications (AICCSA) (pp. 1-5). IEEE.

Nothman, J., et al. (2013). Learning multilingual named entity recognition from Wikipedia. Artificial Intelligence, 194, 151-175.

Peters, M. E., et al. (2018). Deep contextualized word representations. arXiv preprint arXiv:1802.05365.

Radford, A., et al. (2018). Improving language understanding by generative pre-training.

Salah, R. E., \& Qadri binti Zakaria, L. (2017). A comparative review of machine learning for Arabic named entity recognition. International Journal on Advanced Science, Engineering and Information Technology, 7(2), 511-518.

Shahina, K. K., et al. (2019, March). A Sequential Labelling Approach for the Named Entity Recognition in Arabic Language Using Deep Learning Algorithms. In 2019 International Conference on Data Science and Communication (IconDSC) (pp. 1-6). IEEE.

Shihadeh, C., \& ünter Neumann, G. (2012, November). ARNE: A tool for named entity recognition from Arabic text. In Fourth Workshop on Computational Approaches to Arabic Script-based Languages (CAASL4), located at the Tenth Biennial Conference of the Association for Machine Translation in the Americas (AMTA) (pp. 24-31).

Vaswani, A., et al. (2017). Attention is all you need. In Advances in neural information processing systems (pp. 5998-6008).

Wu, Y., et al. (2016). Google's neural machine translation system: Bridging the gap between human and machine translation. arXiv preprint arXiv:1609.08144.

Zaghouani, W. (2012). RENAR: A rule-based Arabic named entity recognition system. ACM Transactions on Asian Language Information Processing (TALIP), 11(1), 1-13. 